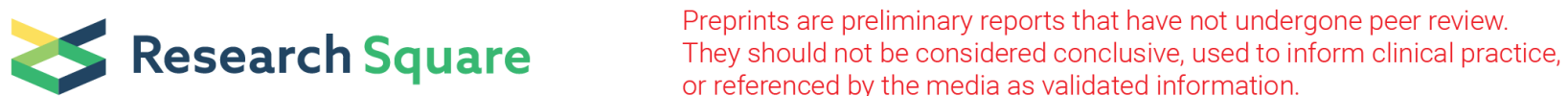 \\ Brain Function in Middle-Aged Hong Chuan Tai Chi Players
}

\section{Zhenguo Shi}

Shandong University

Lei Xu

Shandong University

Hui Xie

Shandong University

Xianliang Zhang

Shandong University

Qiang He ( $\sim$ heqiang224@163.com )

Shandong University

\section{Research Article}

Keywords: Tai Chi, Brain functional connection, Functional near-infrared spectroscopy, Wavelet phase coherence

Posted Date: January 20th, 2021

DOl: https://doi.org/10.21203/rs.3.rs-140252/v1

License: (c) (i) This work is licensed under a Creative Commons Attribution 4.0 International License.

Read Full License 


\title{
Brain Function in Middle-Aged Hong Chuan Tai Chi Players
}

Zhenguo Shi ${ }^{1}$, Lei Xu ${ }^{1}$, Hui Xie ${ }^{2}$, Xianliang Zhang ${ }^{1}$, Qiang $\mathrm{He}^{1 *}$

1. School of Physical Education, Shandong university, 17923 Jingshi Road, Lixia District, Jinan, Shandong Province, China

2. School of Biological Science and Medical Engineering, Beihang University, 37 Xueyuan Road, Haidian District, Beijing, China

*Correspondence: heqiang224@163.com

\begin{abstract}
Background: Tai Chi has been proved as an effective strategy to improve cognitive function while the mechanism remains unclear. This cross-sectional study aimed to compare the brain functional connection of prefrontal cortex (PFC), motor cortex (MC) and occipital cortex (OC) in middle-aged Tai Chi practitioners and Tai Chi-naïve controls.
\end{abstract}

Methods: 18 Hong chuan Tai Chi practitioners (age:55.78 $\pm 2.64 \mathrm{y}$ ) and 22 demographically matched healthy Tai Chi-naïve controls (age:54.69 $\pm 3.10 y$ ) were recruited in this study. Global cognition was measured by the Montreal Cognitive Assessment Scale (MoCA), and the functional connection between PFC, MC and OC in five frequency intervals (I, 0.6-2Hz; II, 0.145-0.6Hz; III, 0.052-0.145Hz; IV, 0.021$0.052 \mathrm{~Hz} ; \mathrm{V}, 0.0095-0.021 \mathrm{~Hz}$ ) was analyzed by wavelet phase coherence (WPCO). The changes in cerebral oxygenation $\left(\Delta\left[\mathrm{HbO}_{2}\right]\right)$ were measured by functional near-infrared spectroscopy (fNIRS). Continuous recordings of NIRS signals were obtained from the left and right prefrontal cortex (PFC), motor cortex (MC) and occipital cortex (OC) in resting state.

Results: Compared with age-matched Tai Chi-naïve controls, Hong chuan Tai Chi practitioners had better global cognition and showed higher functional connection levels between left and right PFC, MC, OC in intervals I, III, VI and V in resting state. Conclusion: This study showed that middle-aged Hong chuan Tai Chi practitioners had higher functional connection between PFC, MC and OC, as well as the coordination of left and right brain in resting state, which maybe the contributing factors to higher global cognition. 
Key words: Tai Chi, Brain functional connection; Functional near-infrared spectroscopy; Wavelet phase coherence

\section{Introduction}

It's well known that aging is a complex degenerative process in brain structure and function, accompanied by cognitive impairment such as decline of learning ability and memory. The degenerative process seriously affects physical health and independent living ability of older adults ${ }^{1}$. How to delay aging-related decline of cognitive function now becomes an important issue to address. Tai Chi, as a form of mind-body exercise originated from ancient China, showed benefits for cognitive function in healthy older adults $^{2,3}$, as well as in older adults with neurodegenerative disease ${ }^{4}$. Tai Chi involves use of slow breath and graceful movement to enhance relaxation and mindfulness. It requires practitioners to control and move their bodies consciously, so that they can achieve unity of body and mind. Skillful completion of Tai Chi requires the coordination of eyes, hands and feet, regulated by different cerebral regions. A metaanalysis involving 2553 participants showed that Tai Chi can increase the strength, balance, attention and cognitive function of practitioners aged 60 or older ${ }^{5}$. Another meta-analysis assessed the effects of mind-body exercise on cognitive performance of adults age ranged from 50-85 and subgroup analysis showed Tai Chi is beneficial for improving global cognition ${ }^{6}$. However, the underlying mechanism remains unclear. Accumulated evidence indicated that such mind-body integration has been proven to reshape the patterns of brain structure, enhance the functional connections between different brain cerebral regions and functional specialization ${ }^{7-9}$.

The neural activity of specific cortical and subcortical regions connects to other brain regions to form functional networks, which is closely associated with cognition behavior. Brain functional connectivity, which refers to the dynamic synchronization of neural activity signals between brain regions, can effectively reflect the synergy of different brain regions ${ }^{10}$. Brain functional connectivity can be assessed not only by functional magnetic resonance imaging ( $\mathrm{AMRI}$ ), electroencephalogram (EEG), but also by functional near-infrared spectroscopy technology (fNIRS) ${ }^{10}$. fNIRS is a noninvasive brain function imaging technology widely used in brain function research in recent years, which can continuously monitor human brain activity by detecting the 
absorption of oxyhemoglobin $\left(\mathrm{HbO}_{2}\right)$ and deoxyhemoglobin $(\mathrm{HHb})$. Compared with fMRI and EEG, fNIRS has high spatial-temporal sampling rate and long data acquisition ability. Multichannel fNIRS can measure the $\mathrm{HbO}_{2}$ changes $\left(\Delta\left[\mathrm{HbO}_{2}\right]\right)$ across several brain regions and their temporal correlation, and analyze brain functional connectivity. Based on these advantages, fNIRS is often used to locate the activation of brain regions and monitor the functional connectivity between different brain regions in resting state or task state ${ }^{11}$. The NIRS signals obtained from the cortical regions during the resting state mainly reflect regional hemodynamic fluctuations that originated from spontaneous cortical activity ${ }^{12}$.

Spontaneous fluctuation of brain activity exists in the absence of overt tasks or stimuli ${ }^{13}$. This spontaneous oscillations are detectable in blood oxygenation level-dependent (BOLD) signals measured by $\mathrm{fMRI}{ }^{13,14}$ and hemoglobin oxygenation signals measured by $\mathrm{fNIRS}^{15-17}$. The power spectra of cerebral $\Delta\left[\mathrm{HbO}_{2}\right]$ exists oscillations in various frequency bands. And the $\Delta\left[\mathrm{HbO}_{2}\right]$ signals in different frequency, which indicates possible regulatory mechanisms of tissue oxygenation signal, have been identified by wavelet analysis $^{18,19}$. The cerebral oscillations in intervals I $(0.6-2 \mathrm{~Hz})$ and II $(0.145-$ $0.6 \mathrm{~Hz}$ ), reflected the synchronization of cardiac and respiratory activities, respectively ${ }^{17}$. The cerebral oscillations in intervals in III $(0.05-0.15 \mathrm{~Hz})$ were suggested to originate locally from intrinsic myogenic activity of smooth muscle cells in resistance vessels and this myogenic mechanism may be partly under autonomic control $^{20}$. Intervals IV $(0.021-0.052 \mathrm{~Hz})$ is closely regulated through tight neurovascular coupling and autonomic control ${ }^{21}$. Intervals $\mathrm{V}(0.0095-0.021 \mathrm{~Hz})$ were regarded as endothelial cell metabolic activities. The spontaneous oscillations in the cerebral hemodynamic signals might be used for monitoring of cerebrovascular pathology and functional activation. Previous studies showed that spontaneous oscillations of cerebral oxygenation in frequency $\mathrm{I}-\mathrm{V}$ in elderly subjects was significantly lower than young subjects ${ }^{19}$. The wavelet phase coherence (WPCO) can be used to determine the possible relationships by evaluating the match between the instantaneous phases of two signals. Wavelet coherence (WCO) identifies high common power while WPCO finds locally phase locked-behavior. WPCO analysis has been used to analyze the relationship 
among oscillations in skin blood flow, blood pressure, brain oxygen saturation within certain specific frequency ranges ${ }^{8,17,18,20,22,23}$.

Robust spatial interactions over the prefrontal, temporal, occipital lobes exist evidenced by evaluating the resting state functional connectivity of whole head using fNIRS ${ }^{8,24}$. Similar results were observed by $\mathrm{fMRI}^{25}$. Prefrontal cortex (PFC), motor cortex (MC) and occipital cortex (OC) are involved in human physical behavior activities. PFC is the cognitive region of human beings, and plays an important role in planning complex cognitive behaviors ${ }^{26}$. MC is located on both sides of the central sulcus of the brain and controls body movement through spatial sensation and motor planning. OC is related to visual perception in motion. The connection between these functional cortexes are important for movement coordination which are required by Tai Chi training. Our previous study reported that the functional connection between these three regions in Tai Chi practitioners was higher than that of Tai Chi-naïve controls in 60 70 years old adults $^{8}$. In addition, there are several forms of Tai Chi, with Chen style the oldest while Yang style the most popular. And most existing studies used these two styles, and reported benefits to brain function ${ }^{8,27}$. Different forms of exercise induce different modulation of brain functional network involved in cognitive control, such as Tai Chi and Baduanjin ${ }^{28}$. And different forms of Tai Chi did induce different changes in terms of brain function ${ }^{27}$. Hong chuan Tai Chi is originated but different from classical Chen style Tai Chi, and is quite popular in Jinan, the capital city of Shandong province, China. As a branch of Chen style Tai Chi, this 81-form Tai Chi evolved with emphasis on art of attach and defence, which is much more strenuous than other forms of Tai Chi. Furthermore, although a previous review of the literature concluded that there was little evidence of cognitive decline before 60 years old ${ }^{29}$. However, it is not universally accepted $^{30}$. A study investigated cognitive decline in five age groups: 45-49y, 50-54y, $55-59 y, 60-64 y$, and 65-70y, the results suggested that cognitive decline is already evident in middle aged (45-49) and increasing with age ${ }^{31}$. And people aged below 60 also suffers decline of cognitive disorders ${ }^{32}$. Moreover, current studies mainly focused on older people $\geqslant 60 \mathrm{y}$ and did not distinguish age groups at all ${ }^{29,33}$. Thus we believe that the effects of Tai Chi practice on brain function of 50-60y middle aged population is worth further exploration. Accordingly, the present study aims to examine Hong 
chuan Tai Chi related changes in brain functional connection in 50-60 years old middleaged adults.

\section{Methods}

\section{Participants}

A total of 40 healthy middle-aged (50 60 years old) adults volunteered and completed this fNIRS study, including 18 Tai Chi practitioners (55.78 \pm 2.64 years old) and 22 demographically matched Tai Chi-naive healthy controls (54.69 \pm 3.10 years old). Control participants were recruited from a local community, with no history of physical exercise, yoga, or mediation treatment. Tai Chi practitioners need to have more than 3 years of Hong chuan Tai Chi experience. On average, the Tai Chi practitioners had 4.61 $(\mathrm{SD}=0.89)$ years of Hongchuan Tai Chi practice experience (Table 1). Subjects with left-handedness, hypertension, history of brain trauma and nervous system diseases, neurological drugs or sleeping pills taken in the past three years, drug abuse were excluded. Before starting the experiment, the subjects were informed of the specific contents of the experiment in advance and gave their written informed consents. The experiment was conducted in accordance with the Declaration of Helsinki, and approved by the ethics committee of rehabilitation hospital affiliated to the National Research Center for Rehabilitation Technical Aids. The ethics committee approved all of the experiment procedures of this study.

\section{Experiment procedures}

The basic information including sex, age, body weight, height, educational background and medical history was recorded by self-reporting, the sleep quality was evaluated by Pittsburgh sleep quality index (PSQI) while global cognitive status was screened by the Montreal Cognitive Assessment Scale (MoCA). Sitting blood pressure were measured by an electronic sphygmomanometer. Each subject was given time to be familiar with the experiment protocol before starting the test. All subjects met the inclusion criteria had a MoCA score of 26 or above, and the PSQI ranged from 1 to 6 . Before starting the fNIRS test, all the subjects are required to maintain a comfortable sitting position, close their eyes and stay awake. After the instrument is placed, all the subjects are required to sit and collect 15 minutes of resting data (without systematic thinking, keep in a relaxed state). A continuous recording of fNIRS signals were then obtained from the 
left and right PFC, MC and OC in 18 Hongchuan Tai Chi practitioners and 22 agematched controls. And the fNIRS measurement was strictly performed by a well-trained technician following the instructions.

\section{Sleep quality}

Before performing the fNIRS test, all the participants accomplished the sleep quality survey in this study. PSQI is a self-rated questionnaire used to evaluate the sleep quality of subjects over a 1-month time interval and has been validated and it was a reliable test for identifying sleep disorders in clinical and research settings. The score is $0-21$ in 19 individual items that generate 7 components. The higher the score is, the worse the sleep quality is, and the score is lower than 6 points, indicating that the sleep quality of subjects is $\operatorname{good}^{34}$.

\section{Global cognitive function}

All the participants completed the global cognitive function test in this study before the fNIRS test using a Chinese version MoCA. MoCA is used to quickly screen mild cognitive impairment, mainly including different cognitive components such as visual space and executive function, memory, attention, language, abstraction etc., with a full score of 30 points. If the number of years of education is less than or equal to 12 years, 1 point will be added, and 26 points or above is considered normal ${ }^{35}$.

\section{Functional near infrared spectroscopy}

A multi-channel tissue oxygenation monitor with continuous-wave (NirSmart, Danyang Huichuang Medical Equipment Co., Ltd) was used in fNIRS measurements. The details had been elaborated in our previous studies ${ }^{8}$. Briefly, each sensor of the instrument consisted of a three-wavelength light emitting diode, which emitted light at 740,808 and $850 \mathrm{~nm}$ wave length, and a detector optode. The 14-channels of the ANIRS were symmetrically positioned over the regions of both left and right PFC, MC and OC. All the protocol was performed by well-trained technician followed the guidelines for Clinical Laboratory Safety. Figure 1 shows the distribution of 14 channels, consisting of 10 light source probes and 8 detector probes, in the brain. The sampling frequency was $10 \mathrm{~Hz}$. 


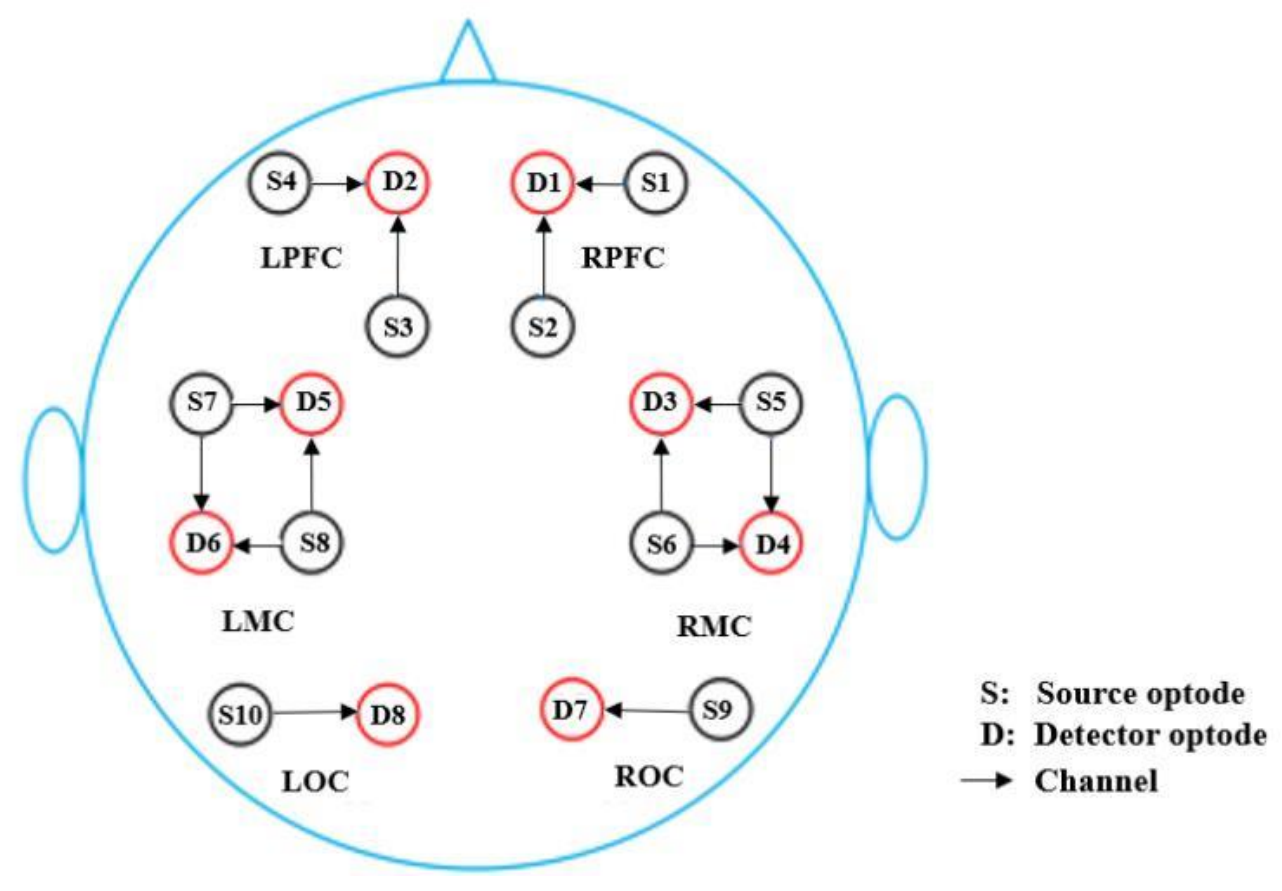

Figure1. Probe arrangement of multichannel blood oxygen detection system. Red circle represents the detector, black circle represents the light source, the arrow is the channel of the light source points to the detector.

\section{Wavelet-based coherence analysis}

The fNIRS signal was always mixed with system physiological noise and tissue noise because some human system physiological activity cannot be stopped, which inevitably affects the hemodynamics, especially in task state. For example, cardiac activity $(0.8 \sim 2 \mathrm{HZ})$, respiration $(0.13 \sim 0.33 \mathrm{HZ})$ can induces global drift of the baseline measurements. In addition, before the near infrared light finally reaches grey matters, it needs to go through tissues including scalp, subcutaneous tissue, skull and cerebrospinal fluid which will influence the fNIRS signal. Finally, the brain spontaneous neural activity might mix cognitive task-induced neuroactivity, like lowfrequency oscillations $(\sim 0.1 \mathrm{HZ})$ and very low-frequency oscillations $(\sim 0.03 \mathrm{HZ})$, although it's can be used in resting state. The aim of data pre-processing was to remove signals with a low signal-to-noise ratio in order to more accurately evaluate brain activity-induced hemodynamics response. To remove these effects, the data can be preprocessed by moving average method for subsequent analysis as described in previous studies ${ }^{36}$. Briefly, MATLAB 2017 was used for data processing and the time window used for moving average filter was 3 seconds. As $\triangle\left[\mathrm{HbO}_{2}\right]$ is more sensitive 
to brain activity, only $\triangle\left[\mathrm{HbO}_{2}\right]$ is used in subsequent signals in this study.

After data pre-processing, continuous wavelet transformation is used to transform the pre-processed data in time-series from the time domain to the time-frequency domain. Then, the fNIRS signals from left and right PFC, MC and OC were analyzed according to five frequency intervals: I, 0.6-2Hz; II, $0.145-0.6 \mathrm{~Hz}$; III, $0.052-0.145 \mathrm{~Hz}$; IV, $0.021-$ $0.052 \mathrm{~Hz} ; \mathrm{V}, 0.0095-0.021 \mathrm{~Hz}$. The physiological meanings of each frequency intervals were: cardiac activity, respiration, vascular smooth muscle activity, neural activity and endothelial cell metabolism activity. Brain functional connection was calculated using the MATLAB 2017 version by methods of wavelet-based phase coherence (WPCO). The wavelet phase coherence is used to detect the correlation degree of two signals at a certain frequency interval. The value of wavelet coherence is between $0-1$ and reflect the instantaneous phase difference between $\triangle \mathrm{HbO}_{2}$. In the analysis of brain functional connection, the higher the wavelet phase coherence value is, the stronger the functional connection between two brain regions indicated by close relationships between two $\triangle$ $\left[\mathrm{HbO}_{2}\right]$ is.

\section{Statistical analysis}

All data were analyzed by SPSS20.0. The results were expressed by mean $\pm \mathrm{sd}$ and the data were processed by independent $T$ test. A significance with $\mathrm{p}<0.05$ was considered statistically significant. Effect size (Cohen's d) and statistical power were also calculated for each dependent variable. Thresholds for effect sizes statistics were: 0.2, trivial; 0.6, small; 1.2, moderate; 2.0, large; and over 2.0, very large.

\section{Results}

\section{Demographic characteristics}

The demographic characteristics of all the participants in each group are analyzed and summarized in Table 1. There were no significant differences $(\mathrm{P}>0.05)$ in age, years of education, BMI , blood pressure, sleep quality between the two groups. Regarding the global cognition determined by MoCA, Hongchuan Tai Chi practitioners demonstrated higher score than healthy Tai Chi-naïve controls $(\mathrm{P}<0.001)$.

Table1. Subjects Characteristics

Tai Chi $(n=18) \quad$ Control $(n=22) \quad$ P




\begin{tabular}{llll}
\hline Age(year) & $55.78 \pm 2.64$ & $54.69 \pm 3.10$ & 0.401 \\
BMI $\left(\mathrm{kg} / \mathrm{m}^{2}\right)$ & $22.10 \pm 0.61$ & $22.27 \pm 0.68$ & 0.556 \\
Education(year) & $12.67 \pm 2.00$ & $12.00 \pm 2.12$ & 0.467 \\
Tai Chi Practice(year) & $4.61 \pm 0.89$ & $\mathrm{NA}$ & $\mathrm{NA}$ \\
Duration(hour/w) & $13.89 \pm 1.62$ & $\mathrm{NA}$ & $\mathrm{NA}$ \\
Right-handed & 18 & 22 & $\mathrm{NA}$ \\
Systolic pressure(mmHg) & $116.00 \pm 7.38$ & $120.85 \pm 6.79$ & 0.128 \\
Diastolic pressure(mmHg) & $73.89 \pm 3.48$ & $76.61 \pm 5.38$ & 0.197 \\
Sleep quality (PQSI) & $3.61 \pm 0.78$ & $3.82 \pm 0.85$ & 0.432 \\
Global cognitive function (MoCA) & $28.06 \pm 0.64$ & $26.27 \pm 1.2$ & 0.000 \\
\hline
\end{tabular}

NA: Not Available

\section{Wavelet phase coherence}

As shown in Fig. 2, compared with Tai Chi-naïve controls, Hong chuan Tai Chi practitioners had higher wavelet phase coherence values of LPFC-LMC(TC $=0.59$, $\mathrm{C}=0.40, \mathrm{t}=4.72, \mathrm{P}<0.001$, Cohen's $\mathrm{d}=1.54$, large $)$ LPFC-LOC(TC $=0.60, \mathrm{C}=0.39$, $\mathrm{t}=5.32, \mathrm{P}<0.001$, Cohen's $\mathrm{d}=1.78$, large), $\mathrm{LMC}-\mathrm{LOC}(\mathrm{TC}=0.53, \mathrm{C}=0.43, \mathrm{t}=2.08, \mathrm{P}<0.05$, Cohen's $\mathrm{d}=0.76$, moderate) in the left hemisphere; and Hong chuan Tai Chi practitioners had higher wavelet phase coherence values of $\mathrm{RPFC}-\mathrm{RMC}(\mathrm{TC}=0.60$, $\mathrm{C}=0.37, \mathrm{t}=5.80, \mathrm{P}<0.001$, Cohen's $\mathrm{d}=1.91$, large $)$ RPFC-ROC(TC=0.55, $\mathrm{C}=0.40$, $\mathrm{t}=3.19, \mathrm{P}<0.01$, Cohen's $\mathrm{d}=1.12$, moderate) in the right hemisphere. In addition, Tai Chi practitioners had higher wavelet phase coherence values of $\mathrm{LPFC}-\mathrm{RPFC}(\mathrm{TC}=0.78$, $\mathrm{C}=0.44, \mathrm{t}=14.35, \mathrm{P}<0.001$, Cohen's $\mathrm{d}=4.67$, very large $), \mathrm{LPFC}-\mathrm{RMC}(\mathrm{TC}=0.60, \mathrm{C}=0.38$, $\mathrm{t}=5.59, \mathrm{P}<0.001$, Cohen's $\mathrm{d}=1.87$, large), LPFC-ROC(TC=0.55, $\mathrm{C}=0.40, \mathrm{t}=3.39$, $\mathrm{P}<0.01$, Cohen's $\mathrm{d}=1.16$, moderate), RPFC-LMC(TC=0.59, $\mathrm{C}=0.39, \mathrm{t}=4.57, \mathrm{P}<0.001$, Cohen's d=1.57, large), RPFC-LOC(TC=0.60, $\mathrm{C}=0.38, \mathrm{t}=5.57, \mathrm{P}<0.001$, Cohen's $\mathrm{d}=1.87$, large), LMC-RMC(TC=0.53, $\mathrm{C}=0.42, \mathrm{t}=2.09, \mathrm{P}<0.05$, Cohen's $\mathrm{d}=0.72$, moderate), LOC-RMC(TC=0.53, $\mathrm{C}=0.42, \mathrm{t}=2.32, \mathrm{P}<0.05$, Cohen's $\mathrm{d}=0.77$, moderate) between the two hemispheres than those of Tai Chi-naïve controls. 

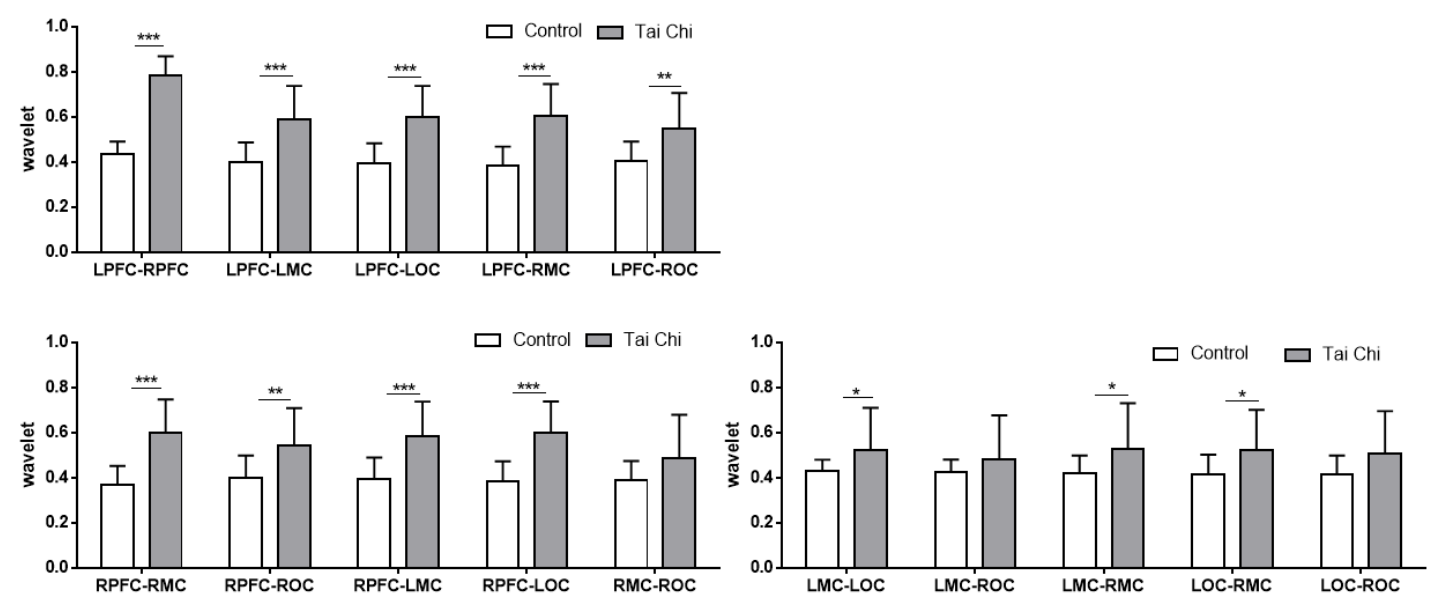

Figure. 2 The wavelet phase coherence values between brain regions in frequency interval $\mathrm{I}$ in resting state. $*(\mathrm{P}<0.05), * *(\mathrm{P}<0.01)$ and $* * *(\mathrm{P}<0.001)$ indicate significant difference between the Tai Chi and Control groups.

Figure. 3 shows the difference of wavelet phase coherence values between the Tai Chi group and the Control group in resting state in interval II. Compared with Tai Chi-naïve controls, Tai Chi practitioners had higher wavelet phase coherence values of LPFCRPFC (TC=0.48, $\mathrm{C}=0.38, \mathrm{t}=2.14, \mathrm{P}<0.05$, Cohen's $\mathrm{d}=0.72$, moderate), and there was no significant difference between other brain regions.
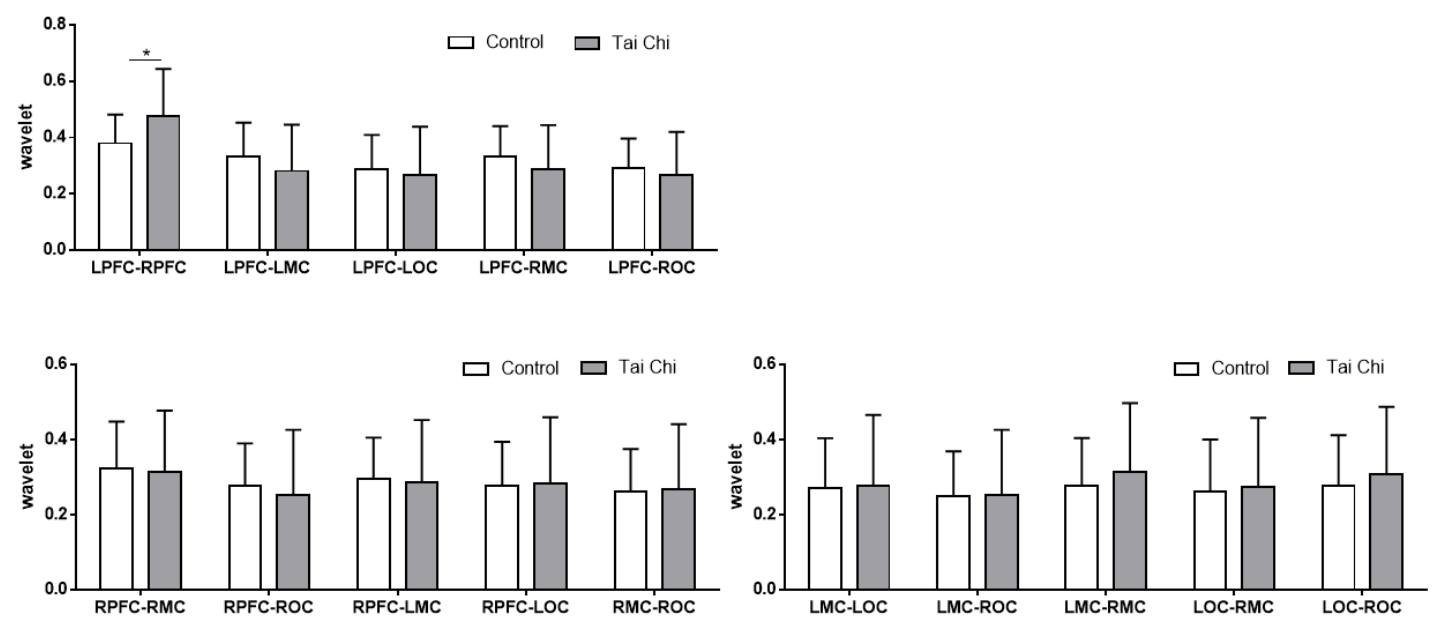

Figure. 3 The wavelet phase coherence values between brain regions in frequency interval II in resting state.

Figure. 4 shows the comparison of wavelet phase coherence values between the Tai Chi group and the Control group in resting state in interval III. Compared with Tai Chi- 
naïve controls, Tai Chi practitioners had higher wavelet phase coherence values of LMC-LOC(TC $=0.47, \mathrm{C}=0.38, \mathrm{t}=2.37, \mathrm{P}<0.05$, Cohen's d=0.76, moderate $)$ and RPFCRMC (TC=0.49, $\mathrm{C}=0.37, \mathrm{t}=3.16, \mathrm{P}<0.01$, Cohen's $\mathrm{d}=1.0$, moderate) in the left and right hemisphere, respectively. In addition, Tai Chi practitioners had higher wavelet phase coherence values of LPFC-RPFC $(\mathrm{TC}=0.63, \mathrm{C}=0.39, \mathrm{t}=7.05, \mathrm{P}<0.001$, Cohen's $\mathrm{d}=2.26$, very large $), \mathrm{LMC}-\mathrm{RMC}(\mathrm{TC}=0.60, \mathrm{C}=0.38, \mathrm{t}=6.05, \mathrm{P}<0.001$, Cohen's $\mathrm{d}=1.99$, large), LOC-RMC ( $\mathrm{TC}=0.47, \mathrm{C}=0.39, \mathrm{t}=2.04, \mathrm{P}<0.05$, Cohen's $\mathrm{d}=0.70$, moderate), LOC-ROC $(\mathrm{TC}=0.50, \mathrm{C}=0.40, \mathrm{t}=2.26, \mathrm{P}<0.05$, Cohen's $\mathrm{d}=0.74$, moderate $)$ between the two hemispheres than Tai Chi-naïve controls.
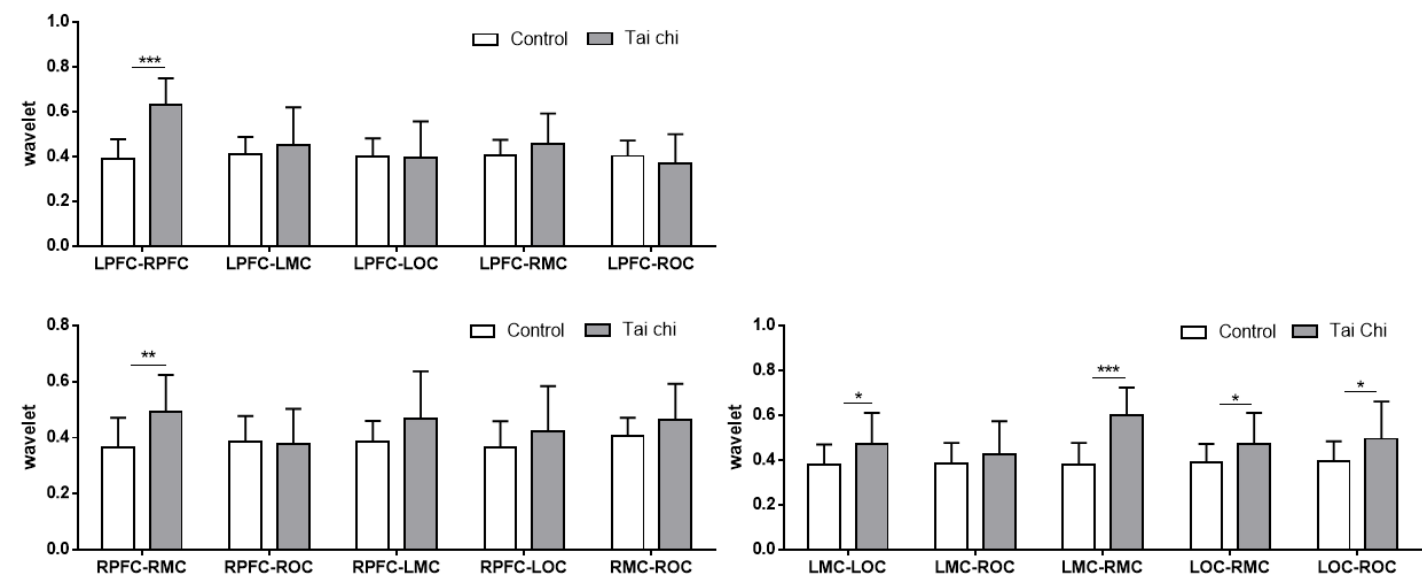

Figure. 4 The wavelet phase coherence values between brain regions in frequency interval II in resting state.

Figure. 5 shows the comparison of wavelet phase coherence values between the Tai Chi group and the Control group in interval IV in resting state. Compared with Tai Chinaïve controls, Tai Chi practitioners had higher wavelet phase coherence values of LPFC-LMC(TC=0.46, $\mathrm{C}=0.37, \mathrm{t}=2.33, \mathrm{P}<0.05$, Cohen's $\mathrm{d}=0.73$, moderate), LMCLOC ( $\mathrm{TC}=0.44, \mathrm{C}=0.36, \mathrm{t}=2.07, \mathrm{P}<0.05$, Cohen's $\mathrm{d}=0.67$, moderate) in the left hemisphere and higher wavelet coherence values of RPFC-RMC $(\mathrm{TC}=0.47, \mathrm{C}=0.36$, $\mathrm{t}=2.61, \mathrm{P}<0.05$, Cohen's $\mathrm{d}=0.90$, moderate) in the right hemisphere. In addition, Tai Chi practitioners had higher wavelet phase coherence values of LPFC-RPFC(TC $=0.59$, $\mathrm{C}=0.37, \mathrm{t}=5.42, \mathrm{P}<0.001$, Cohen's $\mathrm{d}=1.78$, large $), \mathrm{LMC}-\mathrm{RMC}(\mathrm{TC}=0.55, \mathrm{C}=0.36$, $\mathrm{t}=5.39, \mathrm{P}<0.001$, Cohen's $\mathrm{d}=1.76$, large), LOC-RMC(TC=0.43, $\mathrm{C}=0.35, \mathrm{t}=2.29$, $\mathrm{P}<0.05$, Cohen's $\mathrm{d}=0.72$, moderate), LOC-ROC(TC=0.45, $\mathrm{C}=0.36, \mathrm{t}=2.25, \mathrm{P}<0.05$, Cohen's d $=0.76$, moderate) between the two hemispheres compared with Tai Chi-naïve 
controls.
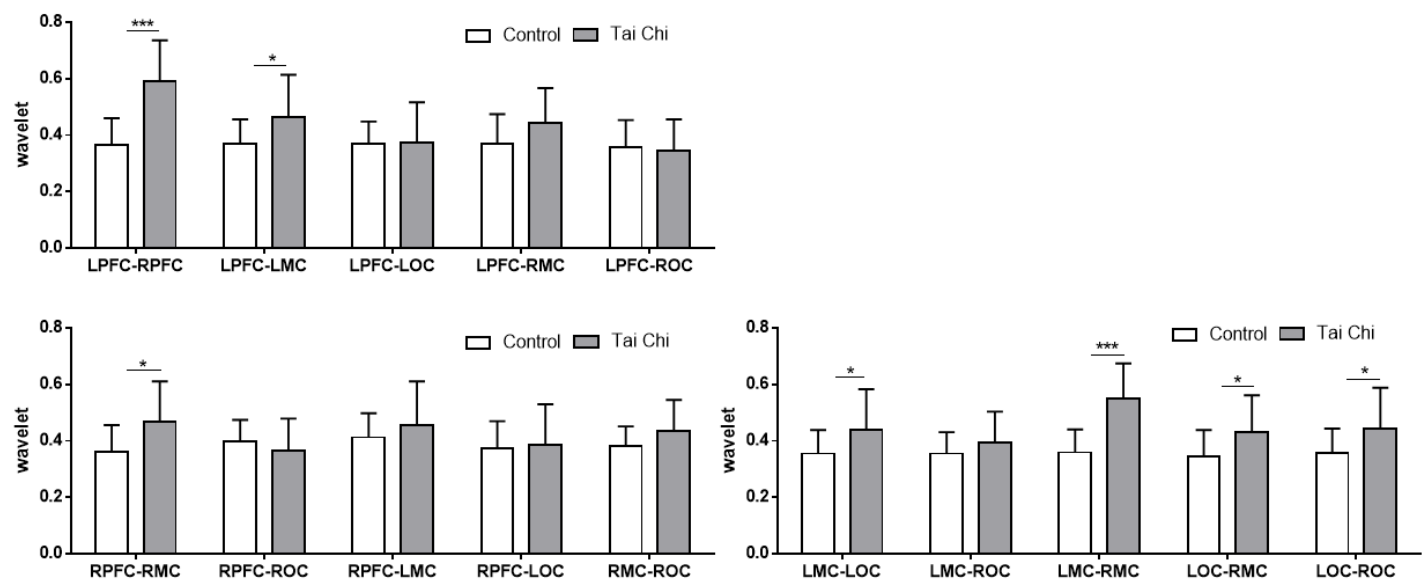

Figure. 5 The wavelet coherence values between brain regions in frequency interval IV in resting state.

Figure. 6 shows the comparison of wavelet phase coherence values between the Tai Chi group and the Control group in interval V in resting state. Compared with Tai Chi-naïve controls, Tai Chi practitioners had higher wavelet phase coherence values of LPFCLMC(TC $=0.48, \mathrm{C}=0.35, \mathrm{t}=3.67, \mathrm{P}<0.01$, Cohen's $\mathrm{d}=1.24$, large $), \mathrm{LMC}-\mathrm{LOC}(\mathrm{TC}=0.48$, $\mathrm{C}=0.38, \mathrm{t}=2.97, \mathrm{P}<0.01$, Cohen's $\mathrm{d}=1.0$, moderate) in the left hemisphere and higher wavelet coherence values of $\mathrm{RPFC}-\mathrm{RMC}(\mathrm{TC}=0.49, \mathrm{C}=0.39, \mathrm{t}=3.26, \mathrm{P}<0.01$, Cohen's $\mathrm{d}=1.04$, moderate), RMC-ROC(TC $=0.48, \mathrm{C}=0.41, \mathrm{t}=2.31, \mathrm{P}<0.05$, Cohen's $\mathrm{d}=0.74$, moderate) in the right hemisphere. In addition, Tai chi practitioners had higher wavelet phase coherence values of LPFC-RPFC $(\mathrm{TC}=0.62, \mathrm{C}=0.36, \mathrm{t}=6.92, \mathrm{P}<0.001$, Cohen's $\mathrm{d}=2.33$, very large), $\mathrm{LPFC}-\mathrm{RMC}(\mathrm{TC}=0.48, \mathrm{C}=0.38, \mathrm{t}=3.25, \mathrm{P}<0.01$, Cohen's $\mathrm{d}=1.11$, moderate), $\mathrm{LMC}-\mathrm{RMC}(\mathrm{TC}=0.55, \mathrm{C}=0.34, \mathrm{t}=6.18, \mathrm{P}<0.001$, Cohen's $\mathrm{d}=2.18$, very large), LOC-RMC(TC=0.48, $\mathrm{C}=0.36, \mathrm{t}=3.61, \mathrm{P}<0.01$, Cohen's $\mathrm{d}=1.19$, moderate $)$, LOC-ROC(TC $=0.53, \mathrm{C}=0.39, \mathrm{t}=3.56, \mathrm{P}<0.01$, Cohen's $\mathrm{d}=1.21$, large) between the two hemispheres than that of Tai Chi-naïve controls. 

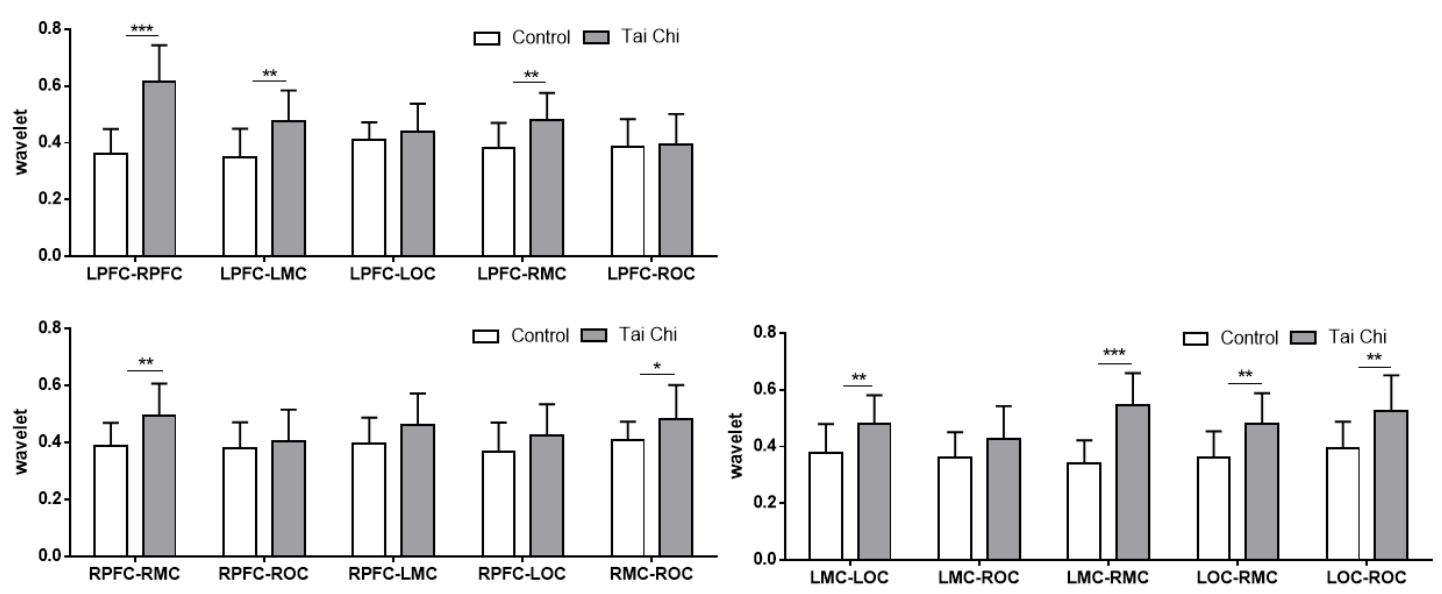

Figure. 6 The wavelet phase coherence values between brain regions in frequency interval $\mathrm{V}$ in resting state.

\section{Discussion}

We designed a cross-sectional study aiming to examine the difference of brain functional connection between Hong chuan Tai Chi practitioners and age-matched Tai Chi-naïve controls. And our results suggested that 50 60 years old Hong chuan Tai Chi practitioners had higher functional connection between PFC, MC and OC, as well as the coordination of left and right brain than age-matched Tai Chi-naïve controls. And this was accompanied with higher global cognitive ability.

Tai Chi is a typical multimodal mind-body exercise that incorporates physical, cognitive, social and meditative components in one activity. Tai Chi might be a prospective strategy to help maintaining cognitive function and delaying cognitive decline of older adults. Previous studies have examined the relationship between longterm Tai Chi exercise and cognitive function in elderly subjects. An early study compared the memory function of older adults aged 56 and older who regularly practiced mind-body or cardiovascular exercises with that of who did not engage in regular exercise, and apparently older adults who practiced Tai Chi and Baduanjin showed better memory function ${ }^{37}$. Another cross-sectional study suggested that the Tai Chi group demonstrated better performance in Mini-mental Status Examination, Color Trail Form attention test, and Rivermead Behavioral Memory Test than other exercise or control groups ${ }^{38}$. In fact, older adults with longer mind body exercise habits had higher scores in most cognitive tests ${ }^{39}$. In current study, we used MoCA to screen the global cognitive ability of 50-60 years old adults. MoCA is a 30 -item test to evaluate 
various domain of cognition like executive function, language, memory, abstraction and orientation. MoCA has been extensively used to assess cognitive impairments in adults in many randomized controlled trials ${ }^{27,40}$. Consistent with previous cross-sectional and cohort studies $^{3,37}$, our study found that Hong chuan Tai Chi practitioners had better global cognitive status than Tai Chi-naïve controls. Although in this study a causal relationship cannot be assumed as many randomized controlled trials did $^{33,41-43}$, it still suggested a close association between Tai Chi practicing and cognitive benefits. A meta-analysis of executive function in randomized controlled trials of cognitively healthy adults indicated a large effect size induced by Tai Chi compared with controls and a moderate effect size compared with other exercise controls ${ }^{5}$.

The changes in brain connectivity might be associated with the decline in cognitive performance ${ }^{44}$. ANIRS is one of the popular non-invasive technology used to measure brain functional connectivity. The intrinsic working principle of brain functional imaging with fNIRS is similar to fMRI, that's the changes of local hemodynamics induced by brain neural activity. The neural activity in different brain cortex needs oxygen and the consumption of oxygen stimulates the local vasodilation and increase blood volume in local capillary which increased cerebral blood flow and cerebral blood volume, also termed functional hyperemia ${ }^{45}$. It's also observed that during cognitive tasks, the $\mathrm{HbO}_{2}$ concentrations in activated brain regions would significantly increase while $\mathrm{HHb}$ levels decrease ${ }^{46}$. While fMRI used the changes of magnetic property induced by hemoglobin changes to get blood oxygen level dependent (BOLD) signal, fNIRS mainly uses the changes of optical characteristics. The main components of human blood including water, $\mathrm{HbO}_{2}$ and $\mathrm{HHb}$, have little absorption of $600-900 \mathrm{~nm}$ wavelength near-infrared light and has good scattering property. In this spectral window, $760 \mathrm{~nm}$ and $850 \mathrm{~nm}$ wavelength near-infrared light have different sensitivity to $\mathrm{HbO}_{2}$ and $\mathrm{HHb}$, respectively. It's believed that the changes of signal attenuation measured during cognitive activity is due to changes of absorption of $\mathrm{HbO}_{2}$ and $\mathrm{HHb}$ in brain. The oxygen and cerebral blood volume can be inferred by measuring the intensity of scattering in that regions. And further changes of $\mathrm{HbO}_{2}\left(\triangle\left[\mathrm{HbO}_{2}\right]\right)$ can be used to evaluate the activation of cognation-related brain regions and the functional connectivity in different regions. Tai Chi is a therapeutic activity strategically to 
improve or maintain brain connectivity in older population. For example, a crosssectional study examined the brain architecture in experienced Tai Chi practitioners and Tai Chi-naïve healthy controls by resting-state fMRI, and found an optimization of the functional organization of brain architectures in older adults with Tai Chi practice history ${ }^{7}$. Another study indicated that Tai Chi evokes significant changes in brain white matter network in older adults ${ }^{47}$. The influence of Tai Chi practice on anatomical connectivity is not discussed in our study. In this study, we used fNIRS to assess the changes of cerebral oxygen signals of middle-aged Hong Chuan Tai Chi practitioners and age-matched healthy Tai Chi-naïve controls to evaluate the functional connection between $\mathrm{PFC}, \mathrm{MC}$ and $\mathrm{OC}$ in resting state at five frequency intervals. The wavelet phase coherence method was extensively used to analyze non-stable signal, and can be used to assess the correlation between two signals with their phase coherence information and evaluates the possible connectivity based on the degree of coincidence of the instantaneous phase over the entire series ${ }^{36}$.

Resting state functional connectivity is a promising neuromarker for cognitive decline in aging population. In our study, a significantly higher wavelet phase coherence values of LPFC-LMC, LPFC-LOC, LMC-LOC, RPFC-RMC, RPFC-ROC and higher wavelet coherence values of LPFC-RPFC, LPFC-RMC, LPFC-ROC, RPFC-LMC, RPFC-LOC, LMC-RMC, LOC-RMC in Hong Chuan Tai Chi practitioners than matched Tai Chinaïve controls were observed in interval I in resting state. Interval I reflects the effect of cardiac activity on cerebral $\triangle \mathrm{HbO}_{2}$. As the cerebrovascular system is a part of cardiovascular system, and with the increase of age, the cardiovascular function of the elderly gradually declines. The result of our study leads to an inference that long-term Hong Chuan Tai Chi practice might improve the cardiac fitness as previous studies reported ${ }^{48}$. And the increased heart pumping function in resting state contributes to the improved functional connection between most brain regions as listed above. The increased functional connection between PFC, MC and OC indicate benefits of Tai Chi on coordination of different brain regions and might be useful to explain the positive relationship between cardiovascular or aerobic fitness and cognition ${ }^{49}$. In addition, the increased wavelet phase coherence in interval I indicated an increased coordinated 
regulation of cardiac activity to cerebral circulation in moving state, and this will increase oxygen supply and nutrients delivery to activated brain regions.

The oscillations in interval II closely resemble the oscillations of respiratory activity ${ }^{8}$ As it usually requires practitioners to coordinate respiration and body movement during Tai Chi exercise. Abdominal respiration used in Tai Chi can increase the intensity of diaphragm relaxation and shrinkage so that it provides adequate oxygen to move body and increase cerebral blood flow, induce changes of cerebral $\triangle \mathrm{HbO}_{2}$. Thus the wavelet phase coherence in interval II reflects the synchronous effect of respiratory activity on $\triangle \mathrm{HbO}_{2}$ in different brain regions. One study suggest that short-term Tai Chi exercise can enhance the pulmonary ventilation function in older adults which may attribute to improved respiratory muscle strength ${ }^{50}$. It's assumed that highly experienced Tai Chi practitioners had better synchronization of breathing and cerebral $\triangle \mathrm{HbO}_{2}$. However, in our study, there's no significant difference in wavelet phase coherence values between most interest brain regions in Tai Chi practitioners and matched controls in resting state, except the LPFC-RPFC connection. It suggested that the oxygen supply of RPFC and LPFC was improved by the breathing of Tai Chi practitioners. A study suggested that the breathing frequency of Tai Chi skilled group was lower than that of the control group, but the breathing depth increased, indicating increased oxygen uptake and cerebral blood oxygen level in resting state ${ }^{51}$. In fact, Tai Chi exercise attach great importance to the coordination of body, mind and breath. The so-called "Qichen Dantian" means deep breathing, which is related to the subconscious control of $\mathrm{PFC}^{52}$.

The oscillations in interval III is associated with myogenic activity of vascular smooth muscle cells which is partially under autonomic control ${ }^{53}$. The contraction and relaxation of vascular smooth muscle directly regulates the diameter and blood vessels pressure. That's the mechanism of myogenic activity influencing cerebral oxygen signals and brain functional connection. Aging is accompanied by a degeneration of vascular system, which may have consequences for cerebral blood flow. Aging induces a reduction of spontaneous very low-frequency $(0.01-0.05 \mathrm{~Hz})$ and low-frequency $(0.07-0.11 \mathrm{~Hz})$ oscillations of cerebral $\mathrm{HbO}_{2}$ and $\mathrm{HHb}$ during both rest and visual stimulation. Another study also found that aging induces a reduction of spontaneous very low-frequency $(0.02-0.07 \mathrm{~Hz})$ and low-frequency $(0.07-0.2 \mathrm{~Hz})$ oscillations of 
cerebral hemodynamics and blood pressure during cognitive task ${ }^{54}$. The declined spontaneous activity in microvascular smooth muscle cells reflects the changes of microvasculature and increase of vessel stiffness. Long-term Tai Chi exercise can improve the autonomic nervous modulation in older adults ${ }^{55}$. It seems that myogenic activity of cerebral vascular smooth muscle cells would be higher in Tai Chi practitioner than that of controls. In this study, Hong chuan Tai Chi practitioners had higher wavelet phase coherence values between LMC-LOC, RPFC-RMC, LPFC-RPFC, LMC-RMC, LOC-RMC, LOC-ROC than those of Tai Chi-naïve controls. This suggests higher functional connection between those regions in highly experienced Tai Chi practitioners and Tai Chi enhanced the synchronization of vascular smooth muscle activities. This further confirms the result reported in our previous studies ${ }^{8}$.

The oscillations in interval IV is related to neurogenic activity, which is regulated by neurovascular coupling and controls smooth muscle cells ${ }^{8}$. The dense synapses on the cerebrovascular can release various types of vasodilator and contractile factors, and most of the vasodilator and diastolic activities of the cerebrovascular are regulated by sympathetic nerve. The robust coupling of neuronal activation to regional cerebral blood flow can be easily observed. For example, the activation of OC by visual stimulation can elicits $\sim 20-30 \%$ increase in blood velocity in the posterior cerebral $\operatorname{arteries}^{56}$. In this study, the wavelet coherence values of LPFC-LMC, LMC-LOC, RPFC-RMC, LPFC-RPFC, LMC-RMC, LOC-RMC, LOC-ROC in Tai Chi practitioners were significantly higher than that of Tai Chi-naïve controls, indicating higher functional connection in resting state after long-term Tai Chi practice. The higher functional connection suggested long-term Tai Chi exercise enhanced the synchronization of neurogenic activity and the coordination of $\mathrm{PFC}, \mathrm{MC}$ and $\mathrm{OC}$ because Tai Chi requires the coordination of movement, mind and visual perception.

The oscillations in interval of $\mathrm{V}$ is associated with the metabolic activity of vascular endothelial cells ${ }^{57}$. Endothelial cells can release potent vasodilator nitric oxide and the nitric oxide -related metabolic activity of endothelial cells is closely integrated with neurovascular activity. Previous studies suggest that Tai Chi can improve endothelial function and arterial stiffness of elderly people with rheumatoid arthritis ${ }^{58}$. It's assumed that Tai Chi can improve metabolic activity of vascular endothelial cells. In this study, 
Hong Chuan Tai Chi practitioners had higher wavelet phase coherence values of LPFCLMC, LMC-LOC, RPFC-RMC, RMC-ROC, LPFC-RPFC, LPFC-RMC, LMC-RMC, LOC-RMC, LOC-ROC than that of Tai chi-naïve controls. This suggests higher functional connection among these brain regions in Tai Chi group.

\section{Conclusion}

Study results from the present study add to growing body of evidence that middle-aged adults with long-term Hong chuan Tai Chi practice experience exhibits higher functional connection between $\mathrm{PFC}, \mathrm{MC}$ and $\mathrm{OC}$, as well as the coordination of left and right brain. And this maybe the underlying mechanism of higher global cognitive function induced by Tai Chi.

\section{Limitations and Future directions}

A cross-sectional study design could not completely rule out the confounding effects of the difference between the two groups of participants, such as lifestyles, environments. In future studies, randomized controlled study may help to rule out these confounding factors and better establish the causal relationship between Hong chuan Tai Chi practice and brain functional connection in several cortex. As the functional connection was performed in the resting state, however, it's hard to judge the mental activity of the Tai Chi practitioners and the matched controls. Tai Chi practitioners might exhibit experienced mediation as Tai Chi required. In the future, we will try to examine the difference of brain functional connection between Tai Chi practitioners and Tai Chinaïve controls in specific cognitive task or exercise. In addition, as Hong chuan Tai Chi is different from classical Chen style Tai Chi, it's necessary to compare their efficiency in brain-related changes in the future.

\section{Abbreviations}

BOLD: blood oxygenation level-dependent; fMRI: functional magnetic resonance imaging; fNIRS: functional near-infrared spectroscopy technology; $\mathrm{HbO}_{2}$ : oxyhemoglobin; $\mathrm{HHb}$ : deoxyhemoglobin; $\mathrm{MC}$ : motor cortex; MoCA: Montreal Cognitive Assessment Scale; OC: occipital cortex; PFC: prefrontal cortex; PSQI: Pittsburgh sleep quality index ; WPCO: wavelet phase coherence

\section{Declarations}

\section{Ethical approval and consent to participate}


This study was performed in accordance with the ethical standards of the 1975 Helsiniki declaration and have been approved by the Human Ethics Committee of National Research Center for Rehabilitation Technical Aids in 2018 (Grant number 20180109). This project is part of the project done by Hui Xie (Tai Chi Chuan exercise related change in brain function as assessed by functional near-infrared spectroscopy) who cooperated with his doctoral supervisor in Beihang university. As there's no ethics committee in our physical education school of Shandong University at that time, so this project was approved by Human Ethics Committee of National Research Center for Rehabilitation Technical Aids. Informed consent was obtained from all individual participants included in this study.

\section{Availability of data and materials}

The datasets used and/or analyzed during the current study available from the corresponding author on reasonable request.

\section{Competing interest}

The authors declare that the research was conducted in the absence of any commercial or financial relationships that could be as a potential conflict of interest.

\section{Funding}

This study was supported by the National Social Science Foundation of China (Grant No: 14BTY027), the Ministry of Education of Humanities and Social Science project (Grant No: 19YJCZH255) and the Fundamental Research Funds of Shandong University (Grant No:2020GN064).

\section{Author Contributions}

ZS, LX, QH contributed to the conception of this work. ZS, HX, XZ and QH designed this study. LX, HX, ZS carried out the experiments. ZS, LX, HX, XZ analyzed the experiments results. ZS, LX, XZ, QH completed the first draft of the manuscript. LX contributed same to ZS to this article and could be considered as common first author. $\mathrm{XZ}$, HX contributed equally with $\mathrm{QH}$ to this article and could be considered as common corresponding author. All authors were involved in the manuscript revision and agreed with final approval of the version, and assured the accuracy of investigation.

\section{Acknowledgments}


The authors sincerely thank all participants who joined the study, thank all cooperating partners and health workers.

\section{References}

1 Anderson ND. Cognitive Neuroscience of Aging. J Gerontol B Psychol Sci Soc Sci. 2019;74:10835.

2 Matthews MM, Williams HG. Can Tai chi enhance cognitive vitality? A preliminary study of cognitive executive control in older adults after A Tai chi intervention. J S C Med Assoc. 2008;104:2557.

$3 \mathrm{Ji} \mathrm{Z,} \mathrm{Li} \mathrm{A,} \mathrm{Feng} \mathrm{T.} \mathrm{The} \mathrm{benefits} \mathrm{of} \mathrm{Tai} \mathrm{Chi} \mathrm{and} \mathrm{brisk} \mathrm{walking} \mathrm{for} \mathrm{cognitive} \mathrm{function} \mathrm{and} \mathrm{fitness} \mathrm{in}$ older adults. PEERJ. 2017;5:e3943.

4 Zhu M, Zhang Y, Pan J. Effect of simplified Tai Chi exercise on relieving symptoms of patients with mild to moderate Parkinson's disease. J Sports Med Phys Fitness. 2020;60:282-8.

5 Wayne PM, Walsh JN, Taylor-Piliae RE. Effect of tai chi on cognitive performance in older adults: systematic review and meta-analysis. J AM GERIATR SOC. 2014;62:25-39.

6 Wu C, Yi Q, Zheng X. Effects of Mind-Body Exercises on Cognitive Function in Older Adults: A Meta-Analysis. J AM GERIATR SOC. 2019;67:749-58.

7 Wei GX, Dong HM, Yang Z. Tai Chi Chuan optimizes the functional organization of the intrinsic human brain architecture in older adults. FRONT AGING NEUROSCI. 2014;6:74.

8 Xie H, Zhang M, Huo C. Tai Chi Chuan exercise related change in brain function as assessed by functional near-infrared spectroscopy. Sci Rep. 2019;9:13198.

9 Chen LZ, Yuan X, Zhang Y. Brain Functional Specialization Is Enhanced Among Tai Chi Chuan Practitioners. Arch Phys Med Rehabil. 2020;101:1176-82.

$10 \mathrm{Niu} \mathrm{H}, \mathrm{He} \mathrm{Y}$. Resting-state functional brain connectivity: lessons from functional near-infrared spectroscopy. NEUROSCIENTIST. 2014;20:173-88.

11 Yeung MK, Chan AS. A Systematic Review of the Application of Functional Near-Infrared Spectroscopy to the Study of Cerebral Hemodynamics in Healthy Aging. NEUROPSYCHOL REV. 2020

12 Sasai S, Homae F, Watanabe H. Frequency-specific functional connectivity in the brain during resting state revealed by NIRS. NEUROIMAGE. 2011;56:252-7.

13 Fox MD, Raichle ME. Spontaneous fluctuations in brain activity observed with functional magnetic resonance imaging. NAT REV NEUROSCI. 2007;8:700-11.

14 Madhyastha TM, Grabowski TJ. Age-related differences in the dynamic architecture of intrinsic networks. Brain Connect. 2014;4:231-41.

15 Li Z, Wang Y, Li Y. Wavelet analysis of cerebral oxygenation signal measured by near infrared spectroscopy in subjects with cerebral infarction. MICROVASC RES. 2010;80:142-7.

16 Cheng R, Shang Y, Hayes DJ. Noninvasive optical evaluation of spontaneous low frequency oscillations in cerebral hemodynamics. NEUROIMAGE. 2012;62:1445-54.

17 Cui R, Zhang M, Li Z. Wavelet coherence analysis of spontaneous oscillations in cerebral tissue oxyhemoglobin concentrations and arterial blood pressure in elderly subjects. MICROVASC RES. 2014;93:14-20.

18 Han Q, Zhang M, Li W. Wavelet coherence analysis of prefrontal tissue oxyhaemoglobin signals as measured using near-infrared spectroscopy in elderly subjects with cerebral infarction. MICROVASC RES. 2014;95:108-15. 
19 Li Z, Zhang M, Xin Q. Age-related changes in spontaneous oscillations assessed by wavelet transform of cerebral oxygenation and arterial blood pressure signals. J Cereb Blood Flow Metab. 2013;33:692-9.

20 Tan Q, Zhang M, Wang Y. Age-related alterations in phase synchronization of oxyhemoglobin concentration changes in prefrontal tissues as measured by near-infrared spectroscopy signals. MICROVASC RES. 2016;103:19-25.

21 Zhang R, Zuckerman JH, Iwasaki K. Autonomic neural control of dynamic cerebral autoregulation in humans. CIRCULATION. 2002;106:1814-20.

$22 \mathrm{Bu} \mathrm{L}$, Li J, Li F. Wavelet coherence analysis of cerebral oxygenation signals measured by nearinfrared spectroscopy in sailors: an exploratory, experimental study. BMJ OPEN. 2016;6:e13357.

23 Tankanag AV, Grinevich AA, Kirilina TV. Wavelet phase coherence analysis of the skin blood flow oscillations in human. MICROVASC RES. 2014;95:53-9.

24 Mesquita RC, Franceschini MA, Boas DA. Resting state functional connectivity of the whole head with near-infrared spectroscopy. BIOMED OPT EXPRESS. 2010;1:324-36.

25 Sakakibara E, Homae F, Kawasaki S. Detection of resting state functional connectivity using partial correlation analysis: A study using multi-distance and whole-head probe near-infrared spectroscopy. NEUROIMAGE. 2016;142:590-601.

26 Miller EK, Cohen JD. An integrative theory of prefrontal cortex function. ANNU REV NEUROSCI. 2001;24:167-202.

27 Zou L, Loprinzi PD, Yu JJ. Superior Effects of Modified Chen-Style Tai Chi versus 24-Style Tai Chi on Cognitive Function, Fitness, and Balance Performance in Adults over 55. Brain Sci. 2019;9

28 Tao J, Chen X, Egorova N. Tai Chi Chuan and Baduanjin practice modulates functional connectivity of the cognitive control network in older adults. Sci Rep. 2017;7:41581.

29 Hedden T, Gabrieli JD. Insights into the ageing mind: a view from cognitive neuroscience. NAT REV NEUROSCI. 2004;5:87-96.

30 Salthouse TA. When does age-related cognitive decline begin? NEUROBIOL AGING. 2009;30:507-14.

31 Singh-Manoux A, Kivimaki M, Glymour MM. Timing of onset of cognitive decline: results from Whitehall II prospective cohort study. BMJ. 2012;344:d7622.

32 Launer LJ. The epidemiologic study of dementia: a life-long quest? NEUROBIOL AGING. 2005;26:335-40.

33 Yang Y, Chen T, Shao M. Effects of Tai Chi Chuan on Inhibitory Control in Elderly Women: An fNIRS Study. FRONT HUM NEUROSCI. 2019;13:476.

34 Buysse DJ, Reynolds CR, Monk TH. The Pittsburgh Sleep Quality Index: a new instrument for psychiatric practice and research. Psychiatry Res. 1989;28:193-213.

35 Nasreddine ZS, Phillips NA, Bedirian V. The Montreal Cognitive Assessment, MoCA: a brief screening tool for mild cognitive impairment. J AM GERIATR SOC. 2005;53:695-9.

$36 \mathrm{Xu} \mathrm{L}$, Wang B, Xu G. Functional connectivity analysis using fNIRS in healthy subjects during prolonged simulated driving. NEUROSCI LETT. 2017;640:21-8.

37 Chan AS, Ho YC, Cheung MC. Association between mind-body and cardiovascular exercises and memory in older adults. J AM GERIATR SOC. 2005;53:1754-60.

38 Man DW, Tsang WW, Hui-Chan CW. Do older t'ai chi practitioners have better attention and 
memory function? J Altern Complement Med. 2010;16:1259-64.

39 Lam LC, Tam CW, Lui VW. Modality of physical exercise and cognitive function in Hong Kong older Chinese community. Int J Geriatr Psychiatry. 2009;24:48-53.

40 Huang N, Li W, Rong X. Effects of a Modified Tai Chi Program on Older People with Mild Dementia: A Randomized Controlled Trial. J ALZHEIMERS DIS. 2019;72:947-56.

41 Sungkarat S, Boripuntakul S, Kumfu S. Tai Chi Improves Cognition and Plasma BDNF in Older Adults With Mild Cognitive Impairment: A Randomized Controlled Trial. Neurorehabil Neural Repair. 2018;32:142-9.

42 Wu MT, Tang PF, Goh J. Task-Switching Performance Improvements After Tai Chi Chuan Training Are Associated With Greater Prefrontal Activation in Older Adults. FRONT AGING NEUROSCI. 2018;10:280.

43 Fong DY, Chi LK, Li F. The benefits of endurance exercise and Tai Chi Chuan for the taskswitching aspect of executive function in older adults: an ERP study. FRONT AGING NEUROSCI. 2014;6:295.

44 Farras-Permanyer L, Mancho-Fora N, Montala-Flaquer M. Age-related changes in resting-state functional connectivity in older adults. NEURAL REGEN RES. 2019;14:1544-55.

45 Nippert AR, Biesecker KR, Newman EA. Mechanisms Mediating Functional Hyperemia in the Brain. NEUROSCIENTIST. 2018;24:73-83.

46 Irani F, Platek SM, Bunce S. Functional near infrared spectroscopy (fNIRS): an emerging neuroimaging technology with important applications for the study of brain disorders. CLIN NEUROPSYCHOL. 2007;21:9-37.

47 Yue C, Zou L, Mei J. Tai Chi Training Evokes Significant Changes in Brain White Matter Network in Older Women. Healthcare (Basel). 2020;8

48 Zheng G, Li S, Huang M. The effect of Tai Chi training on cardiorespiratory fitness in healthy adults: a systematic review and meta-analysis. PLOS ONE. 2015;10:e117360.

49 Hyodo K, Dan I, Kyutoku Y. The association between aerobic fitness and cognitive function in older men mediated by frontal lateralization. NEUROIMAGE. 2016;125:291-300.

50 Song QH, Xu RM, Shen GQ. Influence of Tai Chi exercise cycle on the senile respiratory and cardiovascular circulatory function. INT J CLIN EXP MED. 2014;7:770-4.

51 Quan L.M. (2016). Study on physiological and psychological effects of long term 24-style Tai Chi Chuan exercise on middle-aged people. China Sport Sci \& Tec, 52(05), 68-74.

52 Doll A, Holzel BK, Mulej BS. Mindful attention to breath regulates emotions via increased amygdala-prefrontal cortex connectivity. NEUROIMAGE. 2016;134:305-13.

53 Tan Q, Zhang M, Wang Y. Frequency-specific functional connectivity revealed by wavelet-based coherence analysis in elderly subjects with cerebral infarction using NIRS method. MED PHYS. 2015;42:5391-403.

54 Vermeij A, Meel-van DAA, Kessels RP. Very-low-frequency oscillations of cerebral hemodynamics and blood pressure are affected by aging and cognitive load. NEUROIMAGE. 2014;85 Pt 1:608-15.

55 Lu WA, Kuo CD. The effect of Tai Chi Chuan on the autonomic nervous modulation in older persons. Med Sci Sports Exerc. 2003;35:1972-6.

56 Willie CK, Tzeng YC, Fisher JA. Integrative regulation of human brain blood flow. J Physiol. 
2014;592:841-59.

57 Stewart JM, Taneja I, Goligorsky MS. Noninvasive measure of microvascular nitric oxide function in humans using very low-frequency cutaneous laser Doppler flow spectra. MICROCIRCULATION. 2007; 14:169-80.

58 Shin JH, Lee Y, Kim SG. The beneficial effects of Tai Chi exercise on endothelial function and arterial stiffness in elderly women with rheumatoid arthritis. ARTHRITIS RES THER. 2015;17:380. 


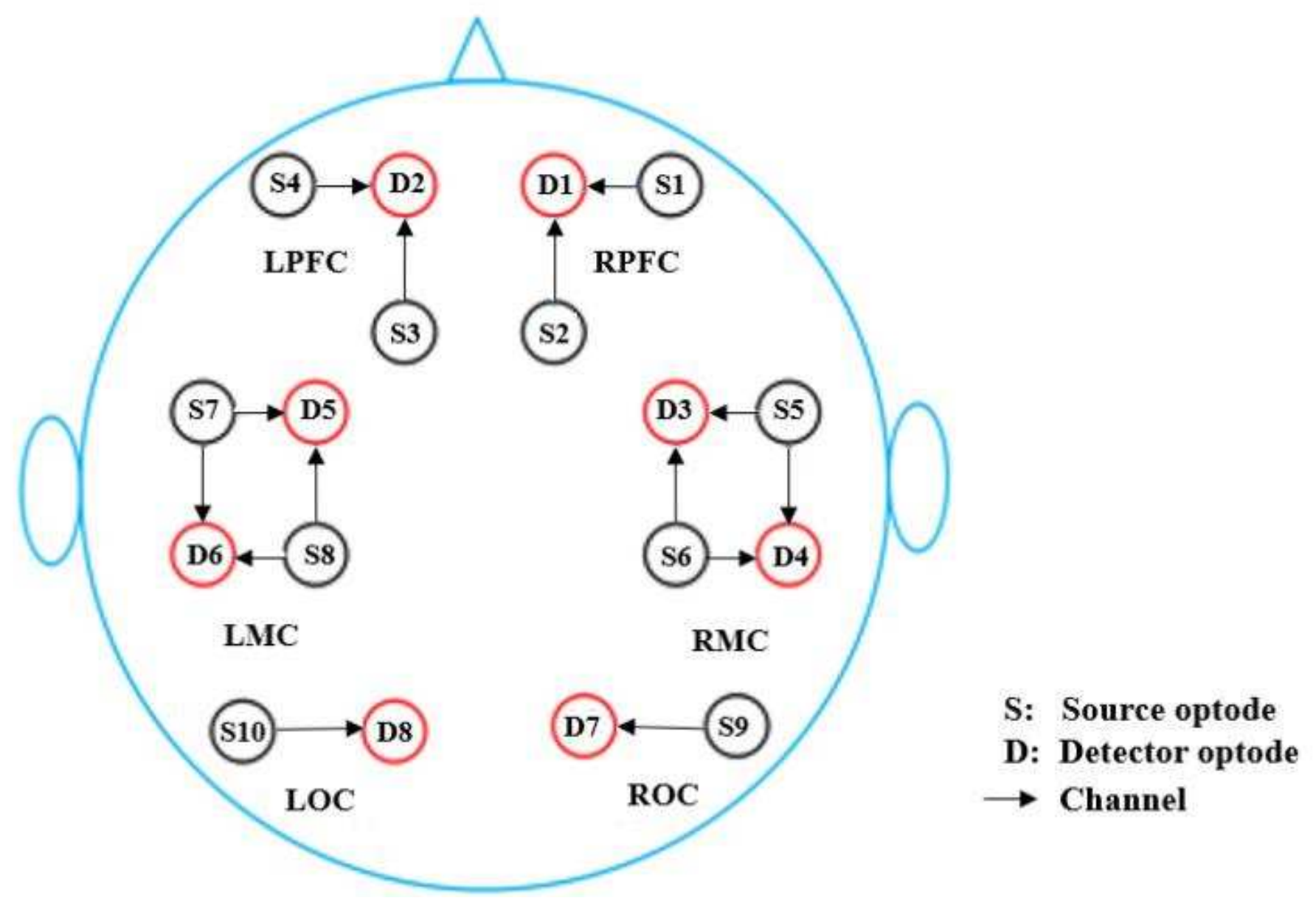

Figure 1

Probe arrangement of multichannel blood oxygen detection system. Red circle represents the detector, black circle represents the light source, the arrow is the channel of the light source points to the detector.
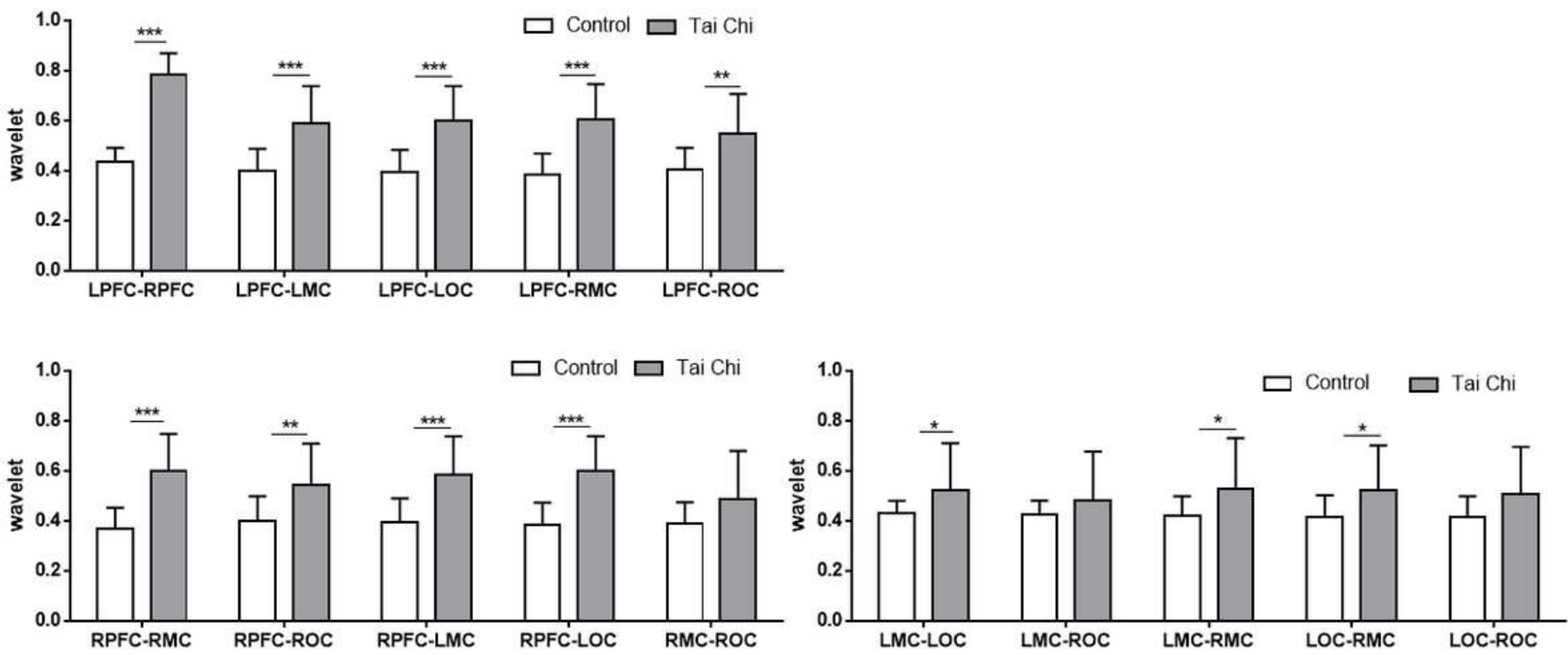
Figure 2

The wavelet phase coherence values between brain regions in frequency interval I in resting state. * $(\mathrm{P} \rrbracket$ $0.05)$, $* \star(P<0.01)$ and $* \star \star(P<0.001)$ indicate significant difference between the Tai Chi and Control groups.
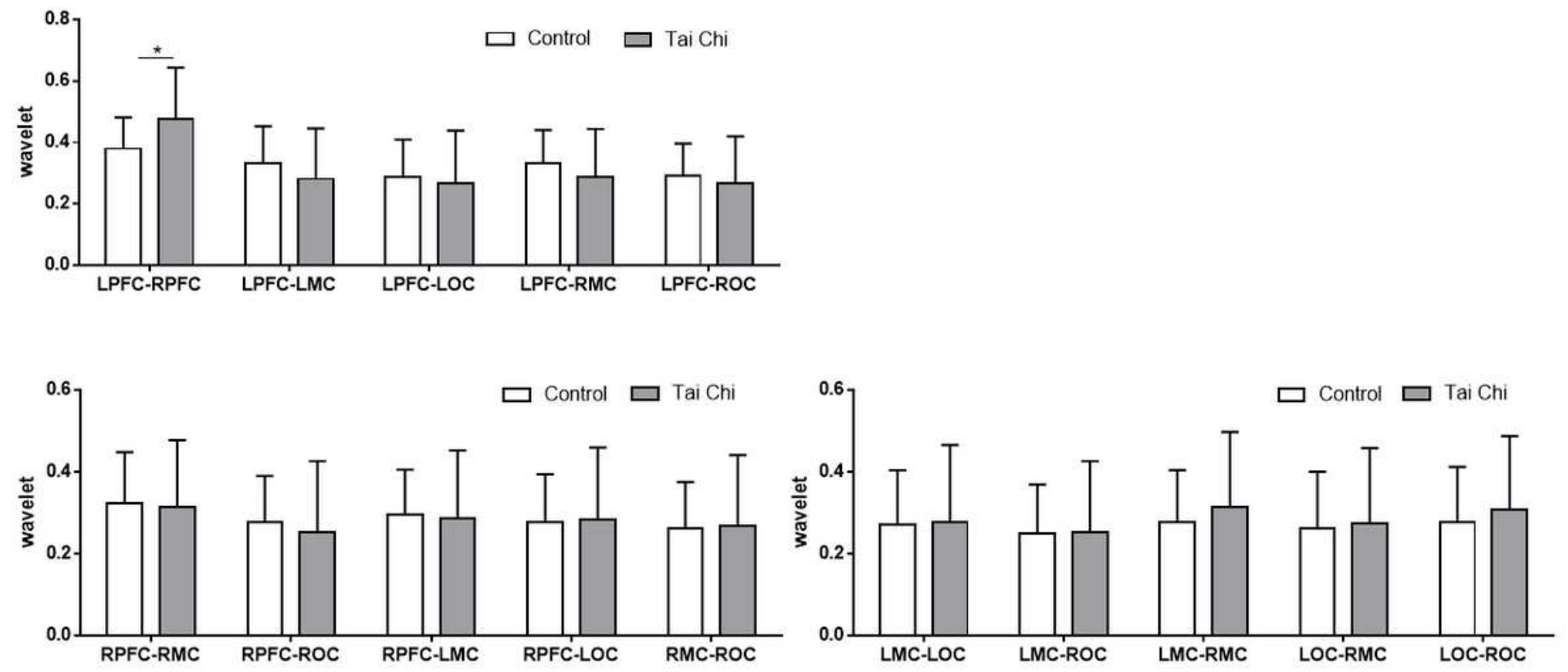

\section{Figure 3}

The wavelet phase coherence values between brain regions in frequency interval II in resting state.
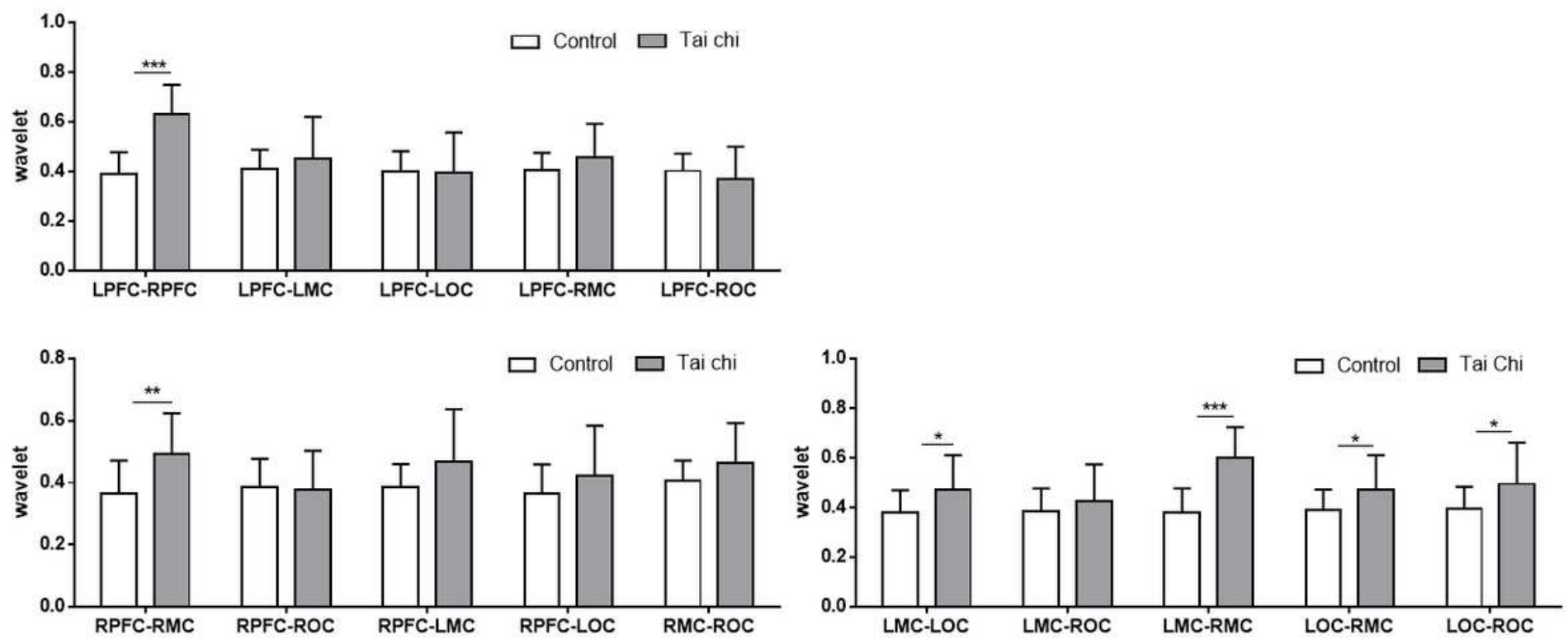

\section{Figure 4}

The wavelet phase coherence values between brain regions in frequency interval III in resting state. 

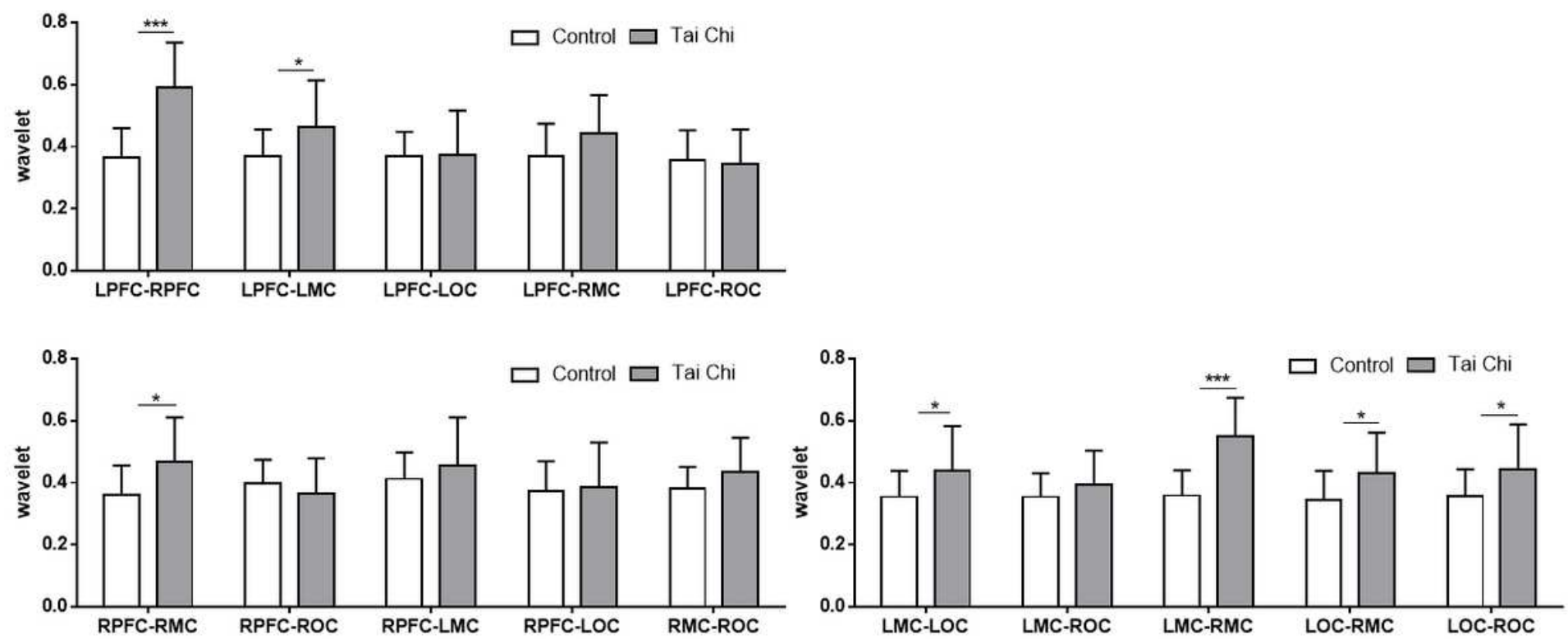

Figure 5

The wavelet coherence values between brain regions in frequency interval IV in resting state.
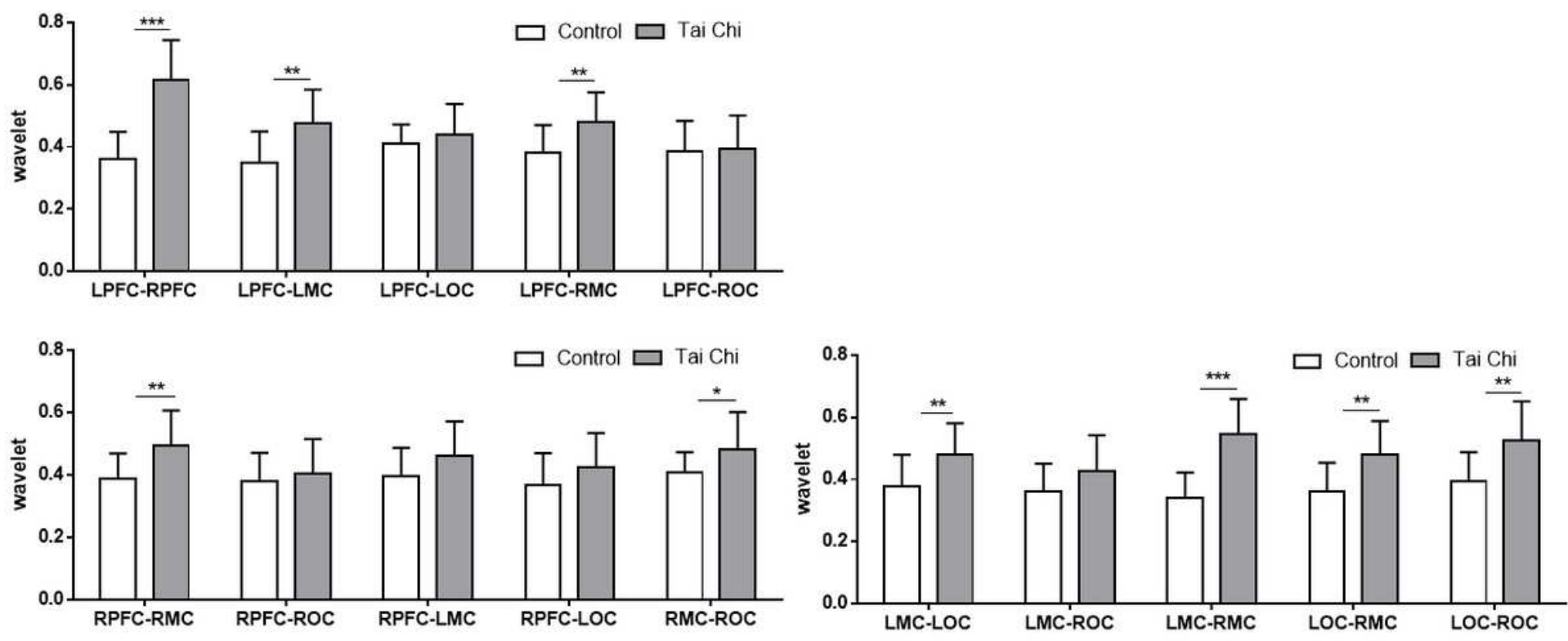

Figure 6

The wavelet phase coherence values between brain regions in frequency interval $\mathrm{V}$ in resting state. 